\section{Grupos vulnerables y su caracterización como criterio de discriminación de la seguridad alimentaria y nutricional en Brasil}

\author{
Vulnerable groups and their \\ characterization like a discrimination \\ criterion of food and nutritional security in \\ Brazil
}

Dixis Figueroa Pedraza 1

1 Programa de Pós-graduação do Departamento de Nutrição. Universidade Federal de Pernambuco. Cidade Universitária Recife, PE, Brasil. CEP: 50. 670-901. E-mail: dixisfigueroa@excite.com

\begin{abstract}
The more exposed people to Food and Nutritional Insecurity comprise the list of the "dispossessed". The most affected families are the ones more vulnerable to environment degradation, living in bad sanitary conditions, contamination and high demographic rates; when this is associated to the maternal and children group vulnerability becomes more accentuated. The quantification of malnourished and undernourished people is as important as determining who they are, where they are, their food habits and the causes and solutions for the problem. Our definition of possible vulnerable groups in Brazil follows two perspectives: poverty related data and FAO's vulnerable group classification (perspectives containing personal criteria, for there are no approaches of the type in scientific literature). High income concentration in Brazil results that the poor have insufficient resources to buy food implying in more vulnerability. There are differences between: urban and rural areas, metropolitan and non-metropolitan areas, and between the Northeast and Northern regions as related to other regions. There are important ethnic, cultural and migration factors involving the more vulnerable groups.
\end{abstract}

Key words Risk groups, Feeding, Malnutrition

\section{Resumen}

Los grupos más expuestos a la Inseguridad Alimentaria y Nutricional son los que figuran en cualquier lista de "desposeídos". Las familias más afectadas son aquellas más vulnerables a la degradación del medio ambiente, que viven en malas condiciones sanitarias, de contaminación y superpoblación; cuando esto se asocia al grupo materno infantil la vulnerabilidad queda reforzada. Si importante es la cantidad de personas con subnutrición y desnutrición, también lo es definir: quiénes son, dónde están, los alimentos que suelen consumir y las causas del problema - soluciones. Nuestra caracterización de los posibles grupos vulnerables en Brasil sigue dos perspectivas: datos relacionados con la pobreza y los criterios para la clasificación de grupos vulnerables de la FAO (perspectiva que refiere criterios personales, pues no existen abordajes de este tipo en la literatura científica). La alta concentración de renta de Brasil hace que los pobres tengan escasos recursos para comprar una cesta básica de alimentos, implicando mayor vulnerabilidad. Se pueden observar diferencias entre: áreas urbanas y rurales, áreas no-metropolitanas y metropolitanas, y entre las regiones nordeste y norte en relación con las demás. Cabe destacar las importantes influencias étnicas, culturales y migratorias que sobre estos grupos de personas pesan y que los hacen vulnerables.

Palabras-claves Grupos vulnerables, Alimentación, Desnutrición 


\section{Introducción}

Los países más susceptibles a tener problemas de inseguridad alimentaria son los que tienen niveles de consumo de alimentos promedio muy bajos, los que presentan grandes oscilaciones en los suministros alimentarios unidos a un bajo nivel de consumo y los que tienen una gran proporción de habitantes pobres. 1

Las características comunes socioeconómicas, agro ecológicas, demográficas y educacionales constituyen factores de vulnerabilidad que operan simultáneamente en combinación para aumentar el riesgo. Las familias más vulnerables a la inseguridad alimentaria son precisamente aquellas más vulnerables a la degradación del medio ambiente, pobres condiciones sanitarias, contaminación y superpoblación. Si nos preguntásemos por qué, a pesar de los adelantos de la ciencia y de la tecnología y de los esfuerzos individuales, de los gobiernos y de organismos en desarrollo, hay aún tanta gente hambrienta sólo pudiéramos respondernos que ello se debe a la inequidad, tanto entre individuos en una misma región o país como entre países. 1,2

Los grupos más expuestos a la inseguridad alimentaria y nutricional son los que figuran en cualquier lista de "desposeídos" : a) campesinos pobres con producción marginal o inadecuada de alimentos; b) núcleos familiares con mujeres al frente; d) núcleos familiares con gran número de integrantes; e) núcleos familiares situados en áreas ecológicamente desventajosas; f) núcleos familiares con ingresos muy bajos para permitir el acceso a suministros de alimentos adecuados en calidad y cantidad.

Es de destacar el grupo materno-infantil como el más vulnerable a los factores que afectan el estado nutricional. Los niños en los primeros años de la vida son los más vulnerables a la inseguridad alimentaria y a los riesgos nutricionales como lo indican las tendencias de mortalidad, de ahí la necesidad de garantizar el acceso a los alimentos. Con determinados niveles de seguridad alimentaria familiar y de higiene del medio algunas familias y niños tienen mejor nutrición que otros. Un elemento fundamental está en la capacidad de las mujeres para prestar cuidados alimentarios, lo cual está en íntima relación con el tiempo disponible, los conocimientos nutricionales, el control de los recursos del hogar y la existencia de determinados bienes de consumo y de servicio. Las mujeres de los hogares pobres son más susceptibles al deterioro de los ingresos y su volumen de trabajo se ve más afectado por situaciones tales como, condiciones precarias de salud, empeoramiento de los precios y dificultad de acceso a los servicios. La lactancia materna es una de las actividades más importantes de la madre para garantizar la seguridad alimentaria. ${ }^{3-6}$

La seguridad alimentaria, además de considerar la disponibilidad y suficiencia de alimentos, debe definir tres grandes grupos como población objeto que deben ser identificados por los sistemas locales de seguridad alimentaria: a) grupos vulnerables que por su condición biológica son más susceptibles a una ingestión deficitaria de alimentos. Estos son: embarazadas, madres que lactan, niños, ancianos; b) grupos en riesgo integrados por la población con dificultades para acceder a los alimentos por su bajo ingreso familiar c) grupos con estado nutricional crítico, población con desnutrición energética crónica.

Identificados los grupos, apoyado en los índices, se toman las decisiones y se evalúa el impacto de estas. Para evaluar los efectos de las políticas y los programas de seguridad alimentaria y monitorear el cambio se necesitan indicadores definidos según el marco político en que se desenvuelven las estrategias de seguridad alimentaria de cada país en cuestión.2,6

\section{Medición de la vulnerabilidad a estados de Inseguridad Alimentaria y Nutricional}

En la lucha contra el hambre mundial, ha surgido un nuevo instrumento para ayudar a las autoridades a orientar sus intervenciones hacia las personas más vulnerables a la inseguridad alimentaria. El "trazado del perfil de los grupos vulnerables"7-9 es un medio de identificar quién padece hambre en una población determinada, por qué y, por deducción, qué se puede hacer al respecto. Este método analítico se ha elaborado como parte de la iniciativa del Sistema de Información y Cartografía sobre la Inseguridad Alimentaria y la Vulnerabilidad (SICIAV). Se puede aplicar sólo o en combinación con otros métodos de evaluación de la vulnerabilidad.

Los resultados pueden ser sorprendentes y dar un fuerte impulso a la actuación. Por ejemplo, en Benin el trazado de un perfil puso de manifiesto que casi la mitad de la población es vulnerable a la inseguridad alimentaria; la Organización de las Naciones Unidas para la Agricultura y la Alimentación (FAO) ${ }^{7,8}$ estima que alrededor de un tercio de esas personas vulnerables ya están subnutridas.

La determinación de los grupos vulnerables en un país requiere numerosas consultas. Un buen punto de partida es una sesión nacional de reflexión 
en la que participe un grupo amplio de interesados, entre ellos representantes de instituciones académicas, asociaciones comerciales, cooperativas, grupos de agricultores, ministerios y organismos oficiales, autoridades locales, organizaciones no gubernamentales, grupos tribales y asociaciones de mujeres.

En Benin se celebró en mayo de 1999 una sesión de este tipo con 40 participantes en representación de todas las regiones y sectores de la sociedad. Los participantes, elegidos por su conocimiento práctico y su experiencia en relación con las condiciones de la seguridad alimentaria en el país, identificaron ocho grupos de subsistencia y tres grupos demográficos vulnerables a la inseguridad alimentaria. En una labor que puede repetirse en todo el mundo, las diversas impresiones y experiencias de los participantes les permitieron identificar:

\section{Quiénes son vulnerables en el país}

Los hogares víctimas de la inseguridad alimentaria pueden pertenecer en distintas zonas a distintos grupos socioeconómicos y demográficos. No obstante, se ponen de manifiesto algunas características comunes de las víctimas de la inseguridad alimentaria, entre las cuales la pobreza y el grupo materno - infantil ocupan un lugar central. Los pobres son quienes se enfrentan a las más rigurosas limitaciones en su propia producción de alimentos y en su acceso a los alimentos en los mercados, lo que los hace vulnerables a las crisis de seguridad alimentaria. Sin embargo, también en hogares con suficiente acceso, pueden tener miembros desnutridos, por causa de la distribución inadecuada de alimentos dentro de la familia, mala salud, hábitos alimentarios inadecuados, cuidados deficientes (especialmente de los niños pequeños) y alimentos de mala calidad e inocuidad. Algunas características sociodemográficas comunes de las familias vulnerables a la inseguridad alimentaria, son las siguientes: a) familias pobres; b) familias numerosas y con un mayor número de miembros dependientes y de menor edad; c) propiedad o acceso limitado a la tierra; d) ingresos bajos de las mujeres; e) falta de diversificación de los ingresos; f) otro aspecto es que en las familias que sufren de inseguridad alimentaria todos los miembros de la familia deben contribuir de alguna manera a las actividades de producción o reproducción relacionadas con la alimentación o nutrición. En esos casos los niños no pueden asistir a la escuela y así ponen en riesgo la seguridad alimentaria a largo plazo. La FAO7-9 desarrolló una metodología (que se explica más abajo) para clasificar y caracterizar a los grupos vulnerables de un país basada en la fuente primaria de sustento, puesto que es fácil de aplicar e integrar los múltiples factores que determinan la inseguridad alimentaria.

\section{Dónde viven}

En general se puede decir que los hogares con riesgo de inseguridad alimentaria viven en áreas donde existe una combinación de varios factores: área con frágil ecosistema, producción baja, aislamiento y acceso limitado a servicios de salud y educación. Además, las crisis pueden exacerbar la vulnerabilidad de los hogares, tales como los desastres naturales (por ejemplo, el huracán Mitch en Centroamérica), guerra, crisis económica o epidemias (por ejemplo el VIH/SIDA);

\section{Los alimentos que suelen consumir}

Los principales alimentos consumidos por una familia o comunidad pueden ser obtenidos por apreciación. El régimen alimenticio se presta particularmente a la observación cualitativa y semicuantitativa por métodos de evaluación rápida. Quienes tienen mayor inseguridad se pueden definir en función de factores como: a) la compra de alimentos más baratos y menos apreciados; b) la compra de solo pequeñas cantidades de alimentos apreciados, como los productos de origen animal. También pueden obtenerse con tales métodos un indicador del número de comidas cocinadas y/o consumidas al día, pero probablemente tiene mayor interés para introducir cambios en el seguimiento.

\section{Los factores que los hacen vulnerables al hambre y la malnutrición}

Identifica las causas del problema y como resolverlo. Hay tantas maneras de clasificar a la población pobre atendiendo a las causas de su hambre (¿quiénes son?). Un sistema particularmente útil de clasificación es la fuente primaria de sustento, puesto que es fácil de aplicar e integra los múltiples factores de los que se deriva la inseguridad alimentaria. Otras bases para la clasificación pueden ser también eficaces, siempre que sean generales y puedan aplicarse en todo el país sin duplicación del recuento. Luego se pueden utilizar los datos del censo y los resultados de otras encuestas para calcular la proporción de la población nacional representada por cada grupo vulnerable. Puede ser necesario desglosar los grupos definidos al principio de manera amplia en distintos subgrupos para garan- 
tizar que las medidas relativas a la seguridad alimentaria sean apropiadas. Cada perfil debe contener información sobre los factores que influyen en el sustento y en consecuencia en la seguridad alimentaria de quienes están incluidos en el perfil. Estos factores son los siguientes: a) diversidad de bienes controlados por los hogares o a los que tienen acceso; b) factores intermedios, como leyes, políticas y reglamentos, que afectan directamente a los hogares, los programas de desarrollo y los proyectos en curso en la zona, y actitudes y creencias locales; c) factores externos, como las tendencias demográficas, la situación de la base de recursos naturales y el rendimiento macroeconómico nacional; d) probabilidad de choques, como descenso de los precios de los productos básicos, sequía, conflictos y enfermedades catastróficas.

Los pescadores artesanales (en pequeña escala) son un ejemplo de un grupo de subsistencia relativamente homogéneo, que es común en todo el mundo y se está convirtiendo cada vez en más vulnerable a la inseguridad alimentaria. Por estos motivos y debido a la disponibilidad de datos de interés, los pescadores artesanales fueron el primer grupo de subsistencia del que se trazó el perfil utilizando el nuevo enfoque del Sistema de Información y Cartografía sobre la Inseguridad Alimentaria y la Vulnerabilidad (SICIAV).

Entre abril y junio de 2000 se realizó un examen general de las condiciones que contribuyen a la vulnerabilidad de este grupo y se elaboraron perfiles de vulnerabilidad para los pescadores artesanales de Benin, Guatemala y Vietnam. Para elaborar estos perfiles, la FAO recurrió a bibliografía secundaria y consultó a personas con conocimiento de las comunidades de pescadores artesanales. En los casos de Guatemala y Vietnam, se realizaron encuestas superficiales para complementar la información.

\section{Caracterización de los grupos alimentariamente vulnerables en Brasil}

Como pudo ser visto en las líneas anteriores el trazado del perfil de los grupos vulnerables es un trabajo complejo y que requiere de un grupo amplio de trabajo. Creemos que en Brasil todavía no existen registros al respecto, para indicar los grupos específicos más vulnerables, como lo existe en otros países como Guatemala. Por esto, para indicar los grupos vulnerables en Brasil seguiremos dos perspectivas: la vulnerabilidad según datos de pobreza, y la vulnerabilidad según criterios para la clasificación de grupos vulnerables de la FAO, uniendo a ello nuestro censo común y conocimientos sobre las características de la población brasileña. Cabe destacar que la segunda perspectiva se refiere a criterios personales, pues no existen abordajes de este tipo en la literatura científica; de esta forma considérense los datos abiertos para el debate, críticas, sugerencias y accesibles a enriquecimientos.

\section{La vulnerabilidad y la pobreza}

Analizando Brasil, como país, sabemos que constituye uno de los países con mayor disponibilidad de alimentos en el mundo, por lo que referido a este aspecto puede considerarse un país con Seguridad Alimentaria, con un número considerable de hogares expuestos a la Inseguridad Alimentaria, caso en el cual los problemas de Seguridad Alimentaria deben ser identificados en general por regiones o grupos socioeconómicos. 10,11

La relación existente entre la pobreza y la inseguridad alimentaria es estrecha y significativa, siendo la pobreza la causa básica y principal de la inseguridad alimentaria. Los grupos vulnerables o en situación de inseguridad alimentaria son aquellos en situación de pobreza y pobreza extrema o en riesgo para esa situación. Comúnmente se usa la renta para estimar la parcela de la población que no tiene condiciones de alimentarse continuamente y que, por tanto, está propicia a pasar hambre. Estar debajo de la línea de pobreza no significa, necesariamente, persona con hambre ya que, por ejemplo, en las áreas rurales familias pobres pueden tener agricultura de subsistencia y no pasar hambre.12,13

Los datos relacionados con la pobreza en Brasil vienen siendo objetivamente publicados en diferentes publicaciones del Instituto de Pesquisa Econômica Aplicada (IPEA). La última publicación del IPEA relacionada con la seguridad alimentaria14 deja claro la grande distancia, que ha permanecido estable en las últimas décadas, entre ricos y pobres de Brasil. Ejemplificando coloca que entre 1977 y 1999, el coeficiente de GINI (mide la concentración de renta), con pocas excepciones, ha permanecido cerca del valor 60 , lo cual se corresponde a uno de los niveles más altos del mundo. En otras palabras, se verifica una simetría social perversa, en que los $10 \%$ más ricos se apropian del $50 \%$ del total de la renta de las familias, y los $50 \%$ más pobres poseen cerca del $10 \%$ de la renta. Además, los $10 \%$ más ricos poseen una renta de 22 a 31 veces encima del valor de la renta obtenida por los $40 \%$ más pobres de la población brasileña.

Entre otros resultados el documento refleja de forma clara la evolución en los indicadores de 
pobreza en Brasil. En 1990 el 44\% de la población residente en Brasil (63 millones de personas) era pobre, valor que bajó en los primeros años de la década y volvió a ser de 44\% en 1994 (67 millones de personas), con el éxito de la estabilización de la economía (Plano Real) la población pobre disminuyó en $10 \%$ y se estabilizó en $33 \%$, lo cual se ha mantenido a pesar de las turbulencias económicas internacionales y locales. En el último año de la década la proporción de pobres se situó en 32\% (54 millones de personas). Por su parte la incidencia de la pobreza extrema o indigencia presentó movimiento semejante: de 21\% (31 millones de personas) en 1990, cayó ligeramente, retornó a $21 \%$ (32 millones) en 1994, cayó para 15\% después del plano real y se fijó en torno del $14 \%$ en los últimos años del período. En el año 2000, la proporción de indigentes atingió $13 \%$ de la población, equivalente a 22 millones de personas. Relacionado a las especificidades (tanto para pobreza como indigencia) se refleja como zonas más afectadas la región Nordeste del país y las áreas urbanas; y resalta los valores significativos en el caso de niños y adolescentes (15 millones abajo de la línea de indigencia en los 4 a 5 primeros años de la década). ${ }^{14}$

Ya en las publicaciones anteriores del IPEA15,16 podemos constatar algunos otros datos importantes $\mathrm{y}$ datos más desagregados. El producto nacional bruto (PIB) per capita ha sido estable (alrededor de USD\$3.500) concluido el período 1985-1989 y 1992-1996. La renta de la familia per cápita bajó entre estos dos períodos (4,2\% por año), pero el porcentaje de las personas que vivían debajo de la línea de la pobreza mostró una disminución moderada ( $1,8 \%$ por año), que se atribuye al aumento leve de la renta de los pobres. Alrededor del $59,1 \%$ de la población es económicamente activa, y de ese segmento, cerca del $7 \%$ fue desempleado en 1996. El desempleo más alto es el de la región centro-oeste $(8,3 \%)$. El desempleo se ha levantado perceptiblemente en los últimos dos años, aunque se espera que el nivel del empleo informal sea alto.

El IPEA también refleja que el grupo de personas (pobres) con más graves problemas de seguridad alimentaria tiene una renta anual alrededor de USD\$170, que es escaso para comprar una cesta básica de alimentos. Aunque el porcentaje de la gente con requisitos básicos insatisfechos disminuyó de $48 \%$ a $22 \%$, estos valores siguen siendo altos. El número total de personas con inseguridad alimentaria se distribuye desigualmente entre las áreas urbanas (metropolitanas y no-metropolitanas) y rurales. Las áreas no-metropolitanas son más afectadas que las metropolitanas: 11,2 y 4,4 millones, respectivamente. La región nordeste concentra el número más alto de indigentes: 17,2 millones. Unos 5,6 millones, de 7,2 millones de personas con Inseguridad Alimentaria de las áreas urbanas, están situados en áreas no-metropolitanas. Las regiones Nordestes y Norte, con el GDP más bajo de 1995, presentan el indicador social peor de Brasil. Las áreas rurales, concentra en el nordeste el número más alto de personas con inseguridad alimentaria (10 millones). La pobreza rural se asocia generalmente a una infraestructura menos desarrollada y a un acceso más bajo a los servicios médicos. En contraste, la región Sudeste es la más rica. Aunque son diferentes en algunos aspectos el sur y el Centro-Oeste, tienen algunas semejanzas con el Sudeste en lo que se refiere a indicadores sociales. Al nivel de estado, Bahía, Minas Gerais y Ceará presentan el número más alto de personas con inseguridad alimentaria y concentran juntas un tercio del número total. Son seguidas por los estados de São Paulo, Pernambuco, Paraná y Río de Janeiro.

\section{La vulnerabilidad siguiendo criterios de la FAO}

Siguiendo los criterios para la clasificación de los grupos vulnerables, segundo Thomson y Metz ${ }^{10} \mathrm{y}$ los sectores de población vulnerables identificados mediante el proceso SICIAV17 y respondiendo a censo común se podrían identificar los siguientes grupos como alimentariamente vulnerables en Brasil:

\section{Geográfico}

Habitantes de zonas urbanas periféricas-marginales, principalmente aquellos que viven en los suburbios urbanos, las llamadas "fabelas" brasileñas. Estas personas, además de no tener un estatus económico favorable, viven en lugares afectados por la falta de organización comunitaria y por condiciones vinculadas a la salud como el escoto sanitario, el acceso a agua potable y la colecta de basura. Dentro de este grupo podemos encontrar una gran variedad de personas que serían clasificadas en el criterio económico.

Habitantes de zonas rurales, principalmente del Norte y Nordeste del país. En el criterio económico deben resaltar los más vulnerables dentro de este grande grupo.

Pescadores artesanales que viven en las zonas litorales del país y en el Norte. Ellos basan su alimentación en el producto de su actividad y muchas veces se da la escasez del pescado, la contaminación de las aguas o la venta a precios bajos. En 
el norte del país se desarrolla la pesca artesanal de ríos y ellos pasan por los mismos inconvenientes que los pescadores de las zonas del litoral.

\section{Ecológico}

Trabajadores rurales que se ven afectados por los períodos de seca o por la estacionalidad de las plantaciones, imposibilitando la estabilidad de la producción. Esto acontece en todo el país pero es más marcado en las zonas "sertão" (parte mayor, más interna y más seca), "agreste" (estrecha facha de transición entre la zona de "mata" y "sertão") y "mata" (zona de clima húmedo, seca verde) del Nordeste del país. El semiárido nordestito ("sertão" + "agreste") es la región más populosa del planeta y además gran parte vive en zona rural, agravando las consecuencias de la seca. Actualmente la única certeza de los estudiosos sobre el tema es que las soluciones para los problemas de la seca solo pueden surgir de la amplia discusión entre los tres niveles del gobierno, los técnicos, representantes de la sociedad civil organizada y la propia población, y que necesitan estar centrados en la idea de convivencia con el semiárido.

Trabajadores rurales del Sur del país que además de ser afectados, aunque en menor medida, por la seca, sus plantaciones se pueden perder por las heladas que acontecen en el invierno.

Trabajadores emigrantes y sus familias o familias a cargo de mujeres de emigrantes. En Brasil existen tres tipos principales de migraciones que acontecen una vez que las personas deciden huir de la pobreza o en la procura de trabajo, y que se acentúa en los períodos de seca. Estos tres tipos de migraciones son: de las zonas "sertão" y "agreste" para los litorales, de las zonas rurales para las zonas urbanas y de las regiones Norte-Nordeste para el Sur-Sudeste. La vulnerabilidad de estas personas se debe a que ellas huyendo de la pobreza o en la búsqueda de trabajo, cuando llegan al lugar de destino se encuentran en lugares que no poseen la infraestructura urbana (agua, escoto, habitación, etc.), social (educación y salud) y económica (oportunidades de empleo, abastecimiento alimentario, etc) para absorberlos y proporcionarles condiciones dignas de vida.

Personas que viven en zonas lejanas de la ciudad y con pocos medios, o ninguno, para llegar hasta ella (inaccesibilidad física).

\section{Económico}

Trabajadores informales que están afectados por los bajos salarios y sin cobertura social en lo referente a seguro de vida, indemnización (por demisión), previdencia social, jubilación, etc. Estos trabajadores pueden encontrarse tanto en las zonas urbanas (donde reciben el nombre de "camelo" o "ambulante") como en las zonas rurales más cercanas de las ciudades (donde reciben el nombre de "boia fria")

Personas con salarios bajos, que no permiten satisfacer sus necesidades básicas y de alimentos. Habitan tanto en las zonas rurales como en las urbanas. La mayor parte de las veces son mano de obra no especializada y con bajo nivel de escolaridad.

Campesinos sin tierra (zonas rurales). Al no tener tierra para trabajar y tampoco renta, se ven imposibilitados de satisfacer sus necesidades básicas y de alimentos.

Trabajadores rurales con tierra pero sin producción, o con muy poca, por la falta de apoyo técnico e institucional.

Personas sin techo - "moradores sem teto" - de zonas urbanas. Son personas que pueden estar empleadas, subempleadas o desempleadas, pero que no tienen donde vivir. Muchos de ellos vienen de las zonas rurales huyendo de la miseria.

Personas sin tierras, conocido en Brasil como "O Grupo dos Sem Terra". Son personas que vivían en el campo como funcionarios, o que perdieron sus tierras y luchan por su tierra propia para producir y para vivir.

Colectores de basura - "coletores de lixo" - que pueden ser "sem teto", "fabelados" o vivir en los basureros - "lixões". Habitan en las zonas urbanas.

Desempleados. Proceden de zonas urbanas y también de las rurales. Al no tener renta, en los mejores casos quedan dependientes de parientes o amigos para subsistir, por lo que son personas dependientes.

Familias encabezadas por mujeres. Cuando la mujer queda como jefe de familia, además de ocuparse con la preparación de los alimentos y cuidado de los menores, tienen que encargarse completamente de las necesidades económicas de la familia. Esto sobrecarga a la mujer y tiende a disminuir significativamente la capacidad de la familia para prestar cuidados a aquellos que lo necesitan, afectando inclusive la lactancia materna. Esta situación la podemos encontrar tanto en las zonas carentes de las zonas urbanas como en las zonas rurales (familias campesinas a cargo de mujeres).

Agricultores de subsistencia o de bajos ingresos de las zonas rurales, incapaces de satisfacer las necesidades familiares de alimentos.

Niños de la calle - "meninos de rua" - y alumnos 
desertores. Estos niños los encontramos principalmente en las zonas urbanas. Ellos se dedican a realizar trabajos en las carreteras (como limpieza de carros, venta de productos baratos) o a pedir dinero, siendo la mayoría de las veces un sustento importante de la familia. Estos niños además de ver imposibilitado su desarrollo normal (físico, biológico, psíquico y social), debido a la realización de tareas no adecuadas para la edad, se ven imposibilitados a una enseñanza y educación adecuada. Los desertores, que por lo general son niños provenientes de familias carentes, además de perder el beneficio del estudio, pierden el beneficio de la merienda escolar; una comida del día garantizada que contribuye a la Seguridad Alimentaria.

Los mendigos que viven en las calles pidiendo dinero para poder subsistir. Son personas de zonas urbanas, afectados en la accesibilidad económica a los alimentos.

Vendedores callejeros, de zonas urbanas, con pocos recursos. Las ganancias que estas personas obtienen de su trabajo no son suficiente para satisfacer sus necesidades.

\section{Demográficos}

Mujeres grávidas o lactantes

Ancianos

Niños de edad preescolar y escolar

Discapacitados y enfermos

Enfermos por HIV

El criterio demográfico agrupa aquellos grupos de personas que biológicamente serían más vulnerables y que precisan de cuidados especiales. Podrían considerarse como condiciones naturales. A nuestro entender, estas personas (que pertenecen a un mismo grupo) podrían diferenciarse indicando una mayor vulnerabilidad para aquellos dependientes, que viven solos o en familia muy numerosa de bajos ingresos. El grupo materno infantil es el que tiene mayor destaque y refuerza su vulnerabilidad cuando se da en conjunto con alguna de las categorías aquí analizadas (geográfico, ecológico, económico, grupo social) e incluso dentro del mismo criterio demográfico.

\section{Grupos sociales en peligro}

Indígenas que están fuertemente amenazados en cuanto a las formas de obtener su sustento de alimentos (caza, pesca, etc.) y combustible, a partir de las zonas forestales donde ellos viven. Esto se hace cada vez más difícil si tenemos en cuenta el aumento progresivo de estas etnias en Brasil y dicho sustento solamente es sostenible cuando la densidad de población, vinculada a él, es baja.

Población (por lo general de raza negra, descendientes de esclavos) que habita las áreas remanecientes de "quilombos" ("quilombolas"), los antiguos refugios de esclavos cuando huían de las haciendas.

Familias analfabetas que no disponen de las condiciones necesarias para tener Seguridad Alimentaria.

Cabe destacar que son cinco las áreas prioritarias (clasificados en alguna de las anteriores categorías) a ser atendidas por el Gobierno Federal brasileño en la implantación del Proyecto "Fome Zero".11,18

Municipios del semiárido del Nordeste, incluido el Valle de Jequitinhonha en Minas Gerais;

Acampamentos y asentamientos rurales;

Población que vive de y en los basureros;

Áreas remanecientes de "quilombos" ("quilombolas") en situación de riesgo nutricional;

Aldeas indígenas en situación de riesgo nutricional.

\section{Conclusiones}

Los factores que influyen en las necesidades nutricionales y en la utilización de los alimentos nos permite identificar los grupos que dentro de una población tienen mayor riesgo de sufrir una deficiencia nutricional. Estos son los llamados grupos vulnerables.

Dentro de cualquier comunidad existen estos grupos de personas que son más sensibles a los cambios de la situación alimentaria, ya que tienen necesidades especiales y/o porque la estructura de la sociedad les impide obtener lo que necesitan. Esto es especialmente válido para los pobres, los analfabetos y los grupos rurales y para el grupo materno infantil, cualquier obstáculo en el sistema de alimentación puede dar como resultado efectos nutricionales adversos en estos grupos vulnerables.

Si importante es la cantidad de personas con subnutrición y desnutrición, cuando abordamos el tema de los grupos vulnerables también se hace importante definir: quiénes son, dónde están, los alimentos que suelen consumir y las causas del problema - soluciones.

La alta concentración de renta de Brasil hace que los pobres tengan escasos recursos para comprar una cesta básica de alimentos, implicando mayor vulne- 
rabilidad. Se observan diferencias entre las áreas urbanas y rurales, las áreas no-metropolitanas (son más afectadas) y metropolitanas y también entre las regiones Nordeste y Norte con relación a las demás.

El trazado del perfil de los grupos vulnerables es un trabajo complejo y que requiere de un grupo amplio de trabajo, inexistente en Brasil. Nuestro instinto nos permitió describir posibles grupos vulnerables en Brasil, que puede ayudar en futuros trabajos y definiciones al respecto. Se destaca las importantes influencias étnicas, culturales y migratorias que sobre estos grupos de personas pesan y que los hacen vulnerables.

\section{Consideraciones finales}

El hambre y la malnutrición siguen siendo los problemas más desbastadores con que se enfrenta la mayoría de la población pobre del mundo. El objetivo de la erradicación del hambre y la malnutrición, y del sufrimiento humano que los acompaña, esta al alcance de la humanidad y sin embargo sigue representando un desafío, por lo que para hacer frente a este reto se hace necesario la acción concertada por parte de todos los interesados: gobiernos, organizaciones no gubernamentales, comunidades locales, el sector privado, la comunidad internacional y las organizaciones internacionales. Las consecuencias de la malnutrición y demás enfermedades nutricionales son variadas y tienen una gran importancia para el bienestar humano y el desarrollo socioeconómico.

La asistencia a grupos socioeconomicamente desfavorecidos y nutricionalmente vulnerables es indispensable para la mejora de su seguridad alimentaria y nutricional. Las políticas para grupos vulnerables (lactantes, niños pequeños, madres, incapacitados, ancianos) deben reforzar la capacidad de la familia, como unidad social y económica, para prestar cuidados y tanto para estos como para los grupos socioeconómicamente desfavorecidos (refugiados, personas desplazadas) es necesario reforzar las capacidades e instituciones en el ámbito comunitario, nacional e internacional.

En muchos hogares las principales personas que prestan cuidados son las mujeres cuya capacidad depende en gran medida de su salud, educación, tiempo, energía, control sobre los recursos del hogar y de su aptitud para utilizarlos eficazmente. La salud física y mental de las madres se promueve facilitando el acceso a los servicios sanitarios y afines y es muy importante difundir directrices dietéticas para las madres durante el embarazo y la lactancia.
El apoyo de la comunidad es decisivo mediante la creación de guarderías infantiles, grupos de trabajo, cooperativas o sistemas oficiosos de reparto de las tareas para reducir el volumen de trabajo, ayudar económicamente, aumentar los conocimientos y brindar apoyo emocional.

A escala nacional los cuidados se refieren a la elaboración de tecnologías apropiadas y crear infraestructura mejor que permita reducir las exigencias de tiempo y trabajo de las mujeres, para las cuales es también necesario reforzar sus derechos, y proporcionarles seguridad social. Las estrategias internacionales para prestar asistencia se aplican cuando los refugiados cruzan las fronteras y los organismos internacionales están llamados a ofrecerles protección social; los refugiados y personas desplazadas necesitan recursos que les ayuden a hacer frente a las privaciones, a cubrir sus necesidades básicas y a ser capaces de valerse por sí mismos.

Adecuadas políticas de población y urbanización son otra pieza clave con relación a la vulnerabilidad alimentaria y nutricional. El crecimiento todavía considerable de la población y la emigración a las ciudades, hace necesaria mayor disponibilidad de alimentos, más bienes, más servicios y más oportunidades de empleo; son varios los problemas económicos, sociales y nutricionales a los que lleva la urbanización y el crecimiento demográfico. Entre las medidas que pueden ser adoptadas se encuentran la promoción de un desarrollo económico más equitativo y las medidas para ofrecer un acceso más fácil a los servicios de educación, salud, y planificación familiar. Los cambios de estilos de vida y en el régimen alimentario asociados a la urbanización conllevan problemas ambientales y con ello enfermedades no transmisibles por lo que debe actuarse al respecto. Otros cambios demográficos importantes que repercuten en la nutrición y que hay que considerar son el aumento del número de personas ancianas ya que ello demanda servicios de salud y sociales debido a la incidencia creciente de enfermedades no transmisibles características de la vejez por lo que resulta evidente la necesidad de promover dietas equilibradas y estilos de vida sanos para impedir estas enfermedades. 


\section{Referencias}

1. Jiménez Acosta S. Algunas consideraciones generales sobre la seguridad alimentaria. III Seminario Internacional de Nutrición; 1994 Ago.; Riobamba, Ecuador.

2. FAO (Organización de las Naciones Unidas para la Agricultura y la Alimentación). El Programa Especial para la Seguridad Alimentaria. Roma; 1996.

3. Britos S. Temas de economía y política alimentaria. Hojas de balance de alimentos como instrumento de análisis de la seguridad alimentaria. Argentina; 2002. Disponible en: http://www.nutrinfo.com.ar. [2002 Dic 14].

4. Ministério da Saúde. Política Nacional de Alimentação e Nutrição. 2. ed. rev. Brasília (DF); 2003

5. Oenema S. La seguridad alimentaria en los hogares. Chile: FAO; 2001.

6. Figueroa Pedraza D. Seguridad alimentaria familiar. Rev Salud Pública Nutr [on line] 2003; 4 (2). Disponible en: www.uanl.mx/publicaciones/respyn [2003 Ago 18]

7. FAO (Organización de las Naciones Unidas para la Agricultura y la Alimentación). Inseguridad alimentaria. La población se ve obligada a convivir con el hambre y teme morir de inanición. El estado de la inseguridad alimentaria en el mundo, 2000. Roma; 2000

8. FAO (Organización de las Naciones Unidas para la Agricultura y la Alimentación). Inseguridad alimentaria. La población se ve obligada a convivir con el hambre y teme morir de inanición. El estado de la inseguridad alimentaria en el mundo, 2001. Roma; 2001.

9. FAO (Organización de las Naciones Unidas para la Agricultura y la Alimentación). Informe de la VIII Mesa Redonda de la Red SISVAN sobre Sistema de Información y Cartografía sobre la Inseguridad Alimentaria y la Vunerabilidad (SICIAV); 1999 Nov 30 al Dic 3; Ciudad de México, México. Disponible en: <http:// www.rlc.fao.org /redes/sisvan/> [2002 Jul 18].
10. Thomson A, Metz M. Implicaciones de las políticas económicas en la seguridad alimentaria: manual de capacitación. Roma: FAO; 1999.

11. Conselho Nacional de Segurança Alimentar e Nutricional. Relatório final da II Conferência Nacional de Segurança Alimentar e Nutricional. A construção da Política Nacional de Segurança Alimentar e Nutricional; 2004 mar 17 - 20; Olinda, Pernambuco. São Paulo: SESC; 2004

12. Hoffmann R. Pobreza, insegurança alimentar e desnutrição no Brasil. Est Avançados 1995; 9: 159-72.

13. Maluf RS, Menezes F, Valente FL. Contribuição ao tema de segurança alimentar no Brasil. Cad Debate 1996; 4: 6688 .

14. IPEA (Instituto de Pesquisa Econômica Aplicada), Ministério das Relações Exteriores. Secretaria de Estado dos Direitos Humanos. A segurança alimentar e nutricional e o direito humano à alimentação. Brasília (DF); 2002

15. Peliano AM. II Balanço das ações de governo no combate à fome e à miséria: versão preliminar para discussão na XI Reunião do Conselho Nacional de Segurança Alimentar e Nutricional. Brasília (DF): IPEA; 1994.

16. Peliano AM. O mapa da fome: subsídios à formulação de uma política de segurança alimentar. Brasília (DF): IPEA; 1993.

17. FAO (Organización de las Naciones Unidas para la Agricultura y la Alimentación). Inseguridad alimentaria. La población se ve obligada a convivir con el hambre y teme morir de inanición. El estado de la inseguridad alimentaria en el mundo, 1999. Roma: FAO; 1999.

18. Instituto Cidadania. Projeto Fome Zero. Uma proposta de política de segurança alimentar para o Brasil; terceira versão. São Paulo: Icidadania; 200

Recebido em 27 de dezembro de 2004

Versão final em 24 de agosto de 2005

Aprovado em 31 de agosto de 2005 